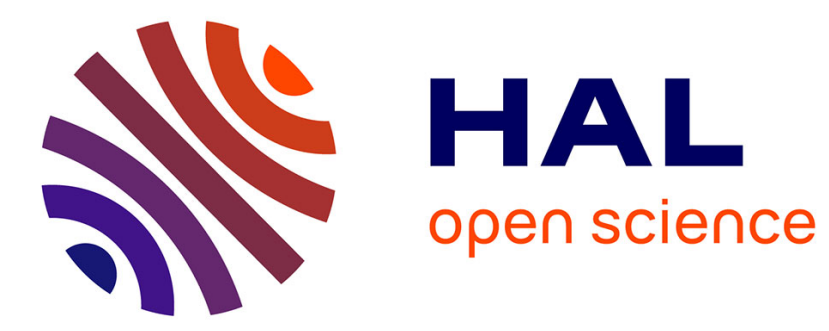

\title{
On enforcing stability for data-driven reduced-order models
}

Ion Victor Gosea, Charles Poussot-Vassal, Athanasios C. Antoulas

\section{To cite this version:}

Ion Victor Gosea, Charles Poussot-Vassal, Athanasios C. Antoulas. On enforcing stability for datadriven reduced-order models. IEEE 29th Mediterranean Conference on Control and Automation MED 2021, Jun 2021, Bari, Italy. hal-03300710

\section{HAL Id: hal-03300710 https://hal.science/hal-03300710}

Submitted on 27 Jul 2021

HAL is a multi-disciplinary open access archive for the deposit and dissemination of scientific research documents, whether they are published or not. The documents may come from teaching and research institutions in France or abroad, or from public or private research centers.
L'archive ouverte pluridisciplinaire HAL, est destinée au dépôt et à la diffusion de documents scientifiques de niveau recherche, publiés ou non, émanant des établissements d'enseignement et de recherche français ou étrangers, des laboratoires publics ou privés. 


\title{
On enforcing stability for data-driven reduced-order models
}

\author{
Ion Victor Gosea ${ }^{1}$, Charles Poussot-Vassal ${ }^{2}$, and Athanasios C. Antoulas ${ }^{3}$
}

\begin{abstract}
In this paper, we address stability enforcement for reduced-order models computed from data (transfer function measurements). Two data-driven methods based on interpolation will be analyzed: the Loewner framework and the AAA algorithm. They construct reduced-order linear models that may or may not be stable. Hence, it is necessary to apply post-processing methods that yield stable surrogate models. We make use of a projection method that computes the best stable approximation with respect to the infinity norm. Finally, we study the applicability and robustness and of the proposed method through different numerical examples.

Index Terms-Data-driven methods, Interpolation-based methods, Loewner matrix, Stable models, Least squares fit.
\end{abstract}

\section{INTRODUCTION}

With the ever-increasing availability of measured data in many engineering and applied science fields, the need for incorporating measurements in the modeling and controlling stage has steadily grown over the last decades. The main challenge consists in using the available data effectively, in order to construct models and controllers that can accurately represent the underlying control system. In this case, the model or controller has to be designed based on experimental measurements, since an exact description (based on equations) is missing. As opposed to model-driven indirect methods, data-driven strategies directly compute the controller from experimental data. Such techniques are also known as direct methods and can be useful when such a control-oriented model is either too complex or too costly to obtain. Consequently, in some applications, a mathematical description of the system is not always available or involves even more complex equations. This is the case when dynamical models are described e.g. by a dedicated simulator, from which the input-output map is not available but can be evaluated. In this second case, instead of relying on equations derived from physical laws, one can infer properties and a model directly from the data, which can be done by means of model approximation, see e.g. [4].

The models under consideration, either obtained from simulations or from finite element/difference methods, might have large dimension (in order to satisfy accuracy requirements). This makes them rather difficult to use for control

\footnotetext{
${ }^{1}$ Ion Victor Gosea is with the Data-Driven System Reduction and Identification (DRI)) Group, Max Planck Institute (MPI), Magdeburg, Germany gosea@mpi-magdeburg.mpg. de

${ }^{2}$ Charles Poussot-Vassal is with the Information Processing and Systems Department, ONERA, Toulouse, France charles.poussot-vassaleonera.fr

${ }^{3}$ Athanasios C. Antoulas is with the Electrical and Computer Engineering (ECE) Department, Rice University, Houston, Max Planck Institute, Magdeburg, Germany and Baylor College of Medicine, Houston, USA acalrice.edu
}

design, numerical simulations or analysis. That is why, it is of critical importance to find reliable reduced-order surrogate models instead. The reduced model may then be used in place of the original one. In this first case, model reduction typically refers to a class of methodologies used for reducing the computational complexity of large-scale models of dynamical systems. Generally, the goal is to approximate the original model with a smaller and simpler one, having the same structure and similar response characteristics as the original. In addition, properties such as stability (or passivity [7]) need to be preserved as well. For an overview of model reduction methods, we refer the reader to the books [3], [8], [4]. In both cases, model reduction and approximation play the pivotal enabler role for model-driven control design.

The scope of this paper is to address stability preservation of reduced-order models computed by means of two methods, i.e., the Loewner framework in [17] and the AAA algorithm in [18]. These are data-driven methods based on interpolation that construct reduced models using only data measurements, e.g. frequency response measurements. One shortcoming of these methods is that they do not necessarily guarantee the stability of the computed models. This represents the motivation for applying post-processing methods, to be able to construct stable models that can be used as surrogates of the original stable systems under consideration. Moreover, the processed models need to approximate well the response of the unprocessed ones (which in turn, are guaranteed to faithfully approximate the underlying dynamics). That is why we apply a projection-based post-processing method that computes best stable approximants with respect to the $\mathcal{H}_{\infty}$-norm (infinity norm), while preserving the rational model dimension. This was originally introduced in [16] and represents a powerful and robust method (see also [22], [23] for applications in aeroelastic aircraft modeling).

The Loewner Framework (LF) is a data-driven model identification and reduction technique. It directly constructs surrogate models that interpolate the data by rearranging the measurements into matrix format, and, potentially reducing its dimension by means of the singular value decomposition. For a tutorial paper on LF for linear systems, we refer the reader to [6]. An extension that uses time-domain data is given in [19], while an extension for certain classes of nonlinear systems, e.g. described by bilinear models, is given in [5]. Finally, enforcing certain properties of the reduced order models (ROMs) computed via LF, such as stability or passivity, were previously studied in [13], [21] (stability), or in [15], [24] (passivity).

The Adaptive-Antoulas-Anderson (AAA) algorithm is a data-driven rational approximation method that combines 
interpolation (as LF) with least squares fitting. AAA yields a reduced-order rational function in barycentric representation that can be interpreted as the transfer function of the surrogate ROM. The AAA algorithm has recently extended for modeling of parametrized dynamics in [9], and for approximation of matrix-valued functions in [14].

The paper is organized as follows: Section II recalls basic properties of linear time-invariant (LTI) systems together with a description of the post-processing method to be utilized. Then, Section III provides a complete review of the two data-driven methods considered here: LF and AAA. Section IV then illustrates the application of the post-processed datadriven methods for two numerical test cases. Finally, the conclusions are provided in Section V.

\section{SET-UP}

\section{A. Preliminary definitions}

We analyze linear time-invariant systems described in generalized state by the following equations,

$$
\boldsymbol{\Sigma}: \mathbf{E} \dot{\mathbf{x}}(t)=\mathbf{A x}(t)+\mathbf{B} u(t), \mathbf{y}(t)=\mathbf{C x}(t)+\mathbf{D} u(t),
$$

where $\mathbf{E}, \mathbf{A} \in \mathbb{R}^{n \times n}, \mathbf{B} \in \mathbb{R}^{n \times m}, \mathbf{C} \in \mathbb{R}^{p \times n}, \mathbf{D} \in$ $\mathbb{R}^{p \times m}, \mathbf{u}(t) \in \mathbb{R}^{m}, \mathbf{y}(t) \in \mathbb{R}^{p}, \mathbf{x}(t) \in \mathbb{R}^{n}$. Denote the class of all these linear systems with $\boldsymbol{\Sigma}_{n, p, m}$. We seek reduced systems of the form

$$
\hat{\boldsymbol{\Sigma}}: \hat{\mathbf{E}} \dot{\hat{x}}(t)=\hat{\mathbf{A}} \hat{\mathbf{x}}(t)+\hat{\mathbf{B}} \mathbf{u}(t), \hat{\mathbf{y}}(t)=\hat{\mathbf{C}} \hat{\mathbf{x}}(t)+\mathbf{D u}(t),
$$

where $\hat{\mathbf{E}}, \hat{\mathbf{A}} \in \mathbb{R}^{r \times r}, \hat{\mathbf{B}} \in \mathbb{R}^{r \times m}, \hat{\mathbf{C}} \in \mathbb{R}^{p \times r}, \hat{x}(t) \in \mathbb{R}^{r}$. The number of inputs and outputs $m$ and $p$, respectively, remain the same, while $r \ll n$. Hence $\hat{\boldsymbol{\Sigma}} \in \boldsymbol{\Sigma}_{r, p, m}$.

Definition 1: Consider the following classes of descriptor linear systems for $m, n, p \in \mathbb{N}$ with $\boldsymbol{\Sigma}_{n, p, m}=$ $\{(\mathbf{E}, \mathbf{A}, \mathbf{B}, \mathbf{C}, \mathbf{D})\}$ where $\mathbf{E}, \mathbf{A} \in \mathbb{R}^{n \times n}, \mathbf{B} \in \mathbb{R}^{n \times m}, \mathbf{C} \in$ $\mathbb{R}^{p \times n}, \mathbf{D} \in \mathbb{R}^{p \times m}$

$\boldsymbol{\Sigma}_{n, p, m}^{0}=\left\{(\mathbf{E}, \mathbf{A}, \mathbf{B}, \mathbf{C}, \mathbf{D}) \in \boldsymbol{\Sigma}_{n, p, m} \mid \rho(\mathbf{A}, \mathbf{E}) \cap i \mathbb{R}=\emptyset\right\}$,

$\boldsymbol{\Sigma}_{n, p, m}^{+}=\left\{(\mathbf{E}, \mathbf{A}, \mathbf{B}, \mathbf{C}, \mathbf{D}) \in \boldsymbol{\Sigma}_{n, p, m} \mid \rho(\mathbf{A}, \mathbf{E}) \subset \mathbb{C}_{+}\right\}$,

$\boldsymbol{\Sigma}_{n, p, m}^{-}=\left\{(\mathbf{E}, \mathbf{A}, \mathbf{B}, \mathbf{C}, \mathbf{D}) \in \boldsymbol{\Sigma}_{n, p, m} \mid \rho(\mathbf{A}, \mathbf{E}) \subset \mathbb{C}_{-}\right\}$,

where $\boldsymbol{\Sigma}_{n, p, m}^{+}$and $\boldsymbol{\Sigma}_{n, p, m}^{-}$are the sets of the antistable and stable systems, respectively. Additionally, $\rho(\mathbf{X}, \mathbf{Y})$ denotes the set of eigenvalues corresponding to the pencil $\xi \mathbf{Y}-\mathbf{X}$, with $\xi \in \mathbb{C}$. Moreover, the matrix $\mathbf{E}$ is considered to be regular (for the class of stable systems).

Definition 2: Let $\boldsymbol{\Sigma}=(\mathbf{E}, \mathbf{A}, \mathbf{B}, \mathbf{C}, \mathbf{D}) \in \boldsymbol{\Sigma}_{n, p, m}$. Then $\mathbf{H}_{\boldsymbol{\Sigma}}: \mathbb{C} \rightarrow \mathbb{C}^{p \times m}$ with $\mathbf{H}_{\boldsymbol{\Sigma}}(s)=\mathbf{C}(s \mathbf{E}-\mathbf{A})^{-1} \mathbf{B}+\mathbf{D}$ is the transfer function of $\boldsymbol{\Sigma}$.

Definition 3: For $i \in\{1,2\}$ and $n_{i} \in \mathbb{N}$, let $\boldsymbol{\Sigma}_{i}=\left(\mathbf{E}_{i}, \mathbf{A}_{i}, \mathbf{B}_{i}, \mathbf{C}_{i}, \mathbf{D}_{i}\right) \in \boldsymbol{\Sigma}_{n_{i}, p, m}$ and

$$
\begin{aligned}
\boldsymbol{\Sigma}_{1} \oplus \boldsymbol{\Sigma}_{2}= & \left(\left[\begin{array}{cc}
\mathbf{E}_{1} & \mathbf{0} \\
\mathbf{0} & \mathbf{E}_{2}
\end{array}\right],\left[\begin{array}{cc}
\mathbf{A}_{1} & \mathbf{0} \\
\mathbf{0} & \mathbf{A}_{2}
\end{array}\right],\left[\begin{array}{l}
\mathbf{B}_{1} \\
\mathbf{B}_{2}
\end{array}\right],\right. \\
& {\left.\left[\begin{array}{ll}
\mathbf{C}_{1} & \mathbf{C}_{2}
\end{array}\right], \mathbf{D}_{1}+\mathbf{D}_{2}\right) . }
\end{aligned}
$$

introduce the following notation $\mathbf{H}_{\boldsymbol{\Sigma}_{1} \oplus \boldsymbol{\Sigma}_{2}}=\mathbf{H}_{\boldsymbol{\Sigma}_{1}}+\mathbf{H}_{\boldsymbol{\Sigma}_{2}}$.
Lemma 1 ([16]): Let $\boldsymbol{\Sigma} \in \boldsymbol{\Sigma}_{n, p, m}^{0}$. Then we can always find $\boldsymbol{\Sigma}_{+}=\left(\mathbf{E}_{+}, \mathbf{A}_{+}, \mathbf{B}_{+}, \mathbf{C}_{+}, \mathbf{D}\right) \in \boldsymbol{\Sigma}_{n_{+}, p, m}^{+}, \boldsymbol{\Sigma}_{-}=$ $\left(\mathbf{E}_{-}, \mathbf{A}_{-}, \mathbf{B}_{-}, \mathbf{C}_{-}, 0\right) \in \boldsymbol{\Sigma}_{n_{-}, p, m}^{-}$so that $\boldsymbol{\Sigma} \sim\left(\boldsymbol{\Sigma}_{+} \oplus \boldsymbol{\Sigma}_{-}\right)$. Proposition 1 ([16]): Consider a linear stable system $\boldsymbol{\Sigma}=(\mathbf{E}, \mathbf{A}, \mathbf{B}, \mathbf{C}, \mathbf{D}) \in \boldsymbol{\Sigma}_{n, p, m}^{-}$. Then, there exist unique and symmetric matrices $\mathcal{P}, \mathcal{Q} \in \mathbb{R}^{n \times n}$ (infinite Gramians) so that the following Lyapunov equations are satisfied

$\mathbf{A} \mathcal{P} \mathbf{E}^{T}+\mathbf{E} \mathcal{P} \mathbf{A}^{T}+\mathbf{B B}^{T}=\mathbf{0}, \mathbf{A}^{T} \mathcal{Q} \mathbf{E}+\mathbf{E}^{T} \mathcal{Q} \mathbf{A}+\mathbf{C}^{T} \mathbf{C}=\mathbf{0}$.

Definition 4: $\mathcal{R} \mathcal{H}_{2}$ and $\mathcal{R} \mathcal{H}_{\infty}$ are the spaces of real rational functions in the Hardy spaces $\mathcal{H}_{2}$ and $\mathcal{H}_{\infty}$, respectively.

\section{B. Post processing method}

In this section we present the post-processing method originally introduced in [16]. There, given an unstable descriptor linear system as in (1) with transfer function $\mathbf{H}$, it is of interest to find a stable descriptor system whose transfer function is the best approximation in the spaces $\mathcal{R} \mathcal{H}_{2}$ and $\mathcal{R} \mathcal{H}_{\infty}$. The following approximation problem denoted with $\mathrm{AP}_{q}$ is stated below:

Problem 1: Let $q \in\{2, \infty\}$ and $\boldsymbol{\Sigma} \in \boldsymbol{\Sigma}_{n, p, m}^{0}$. We are interested in finding $\hat{\boldsymbol{\Sigma}} \in \cup_{\hat{n} \in \mathbb{N}} \boldsymbol{\Sigma}_{\hat{n}, p, m}^{+}$so that

$$
\left\|\mathbf{H}_{\boldsymbol{\Sigma}}-\mathbf{H}_{\hat{\boldsymbol{\Sigma}}}\right\|_{q}=\inf _{\tilde{\boldsymbol{\Sigma}} \in \cup_{\hat{n} \in \mathbb{N}} \boldsymbol{\Sigma}_{\tilde{n}, p, m}^{+}}\left\|\mathbf{H}_{\boldsymbol{\Sigma}}-\mathbf{H}_{\tilde{\boldsymbol{\Sigma}}}\right\|_{q} .
$$

First, we show that solving $\left(\mathrm{AP}_{q}\right)$ for a descriptor system we can equivalently solve $\left(\mathrm{AP}_{q}\right)$ for its antistable part. The following result was proposed in [16] (Theorem 3.1).

Lemma 2: Let $\boldsymbol{\Sigma} \in \boldsymbol{\Sigma}_{n, p, m}^{0}$. Let $q \in\{2, \infty\}$ and $\gamma \geqslant 0$. Then the following two are equivalent

$$
\begin{gathered}
i) \exists \hat{\boldsymbol{\Sigma}} \in \cup_{\hat{n} \in \mathbb{N}} \boldsymbol{\Sigma}_{\hat{n}, p, m}^{+}:\left\|\mathbf{H}_{\boldsymbol{\Sigma}}-\mathbf{H}_{\hat{\boldsymbol{\Sigma}}}\right\| \geqslant \gamma, \\
i i) \exists \hat{\boldsymbol{\Sigma}} \in \cup_{\hat{n} \in \mathbb{N}} \boldsymbol{\Sigma}_{\hat{n}, p, m}^{+}:\left\|\mathbf{H}_{\boldsymbol{\Sigma}_{-}}-\mathbf{H}_{\hat{\boldsymbol{\Sigma}}}\right\| \geqslant \gamma .
\end{gathered}
$$

If $\hat{\boldsymbol{\Sigma}}$ satisfies ii) then $\hat{\boldsymbol{\Sigma}} \oplus \boldsymbol{\Sigma}_{+}$satisfies $i$ ).

The optimal approximation in the $\mathcal{R H}_{2}$ space is given by the following result from [16].

Lemma 3: Let $\boldsymbol{\Sigma} \in \boldsymbol{\Sigma}_{n, p, m}^{0}$ - then $\boldsymbol{\Sigma}_{+}$solves $\left(\mathrm{AP}_{2}\right)$ i.e.,

$\inf _{\tilde{\boldsymbol{\Sigma}} \in \cup_{\tilde{n} \in \mathbb{N}} \boldsymbol{\Sigma}_{\tilde{n}, p, m}^{+}}\left\|\mathbf{H}_{\boldsymbol{\Sigma}}-\mathbf{H}_{\tilde{\boldsymbol{\Sigma}}}\right\|_{2}=\left\|\mathbf{H}_{\boldsymbol{\Sigma}}-\mathbf{H}_{\boldsymbol{\Sigma}_{+}}\right\|_{2}=\left\|\mathbf{H}_{\boldsymbol{\Sigma}}\right\|_{2}$.

For the case of $\mathcal{R} \mathcal{H}_{\infty}$, the problem becomes more complex. For minimal antistable standard systems, the problem $\left(\mathrm{AP}_{\infty}\right)$, also known as a Nehari problem, was already solved in [12]. The following more general result from [16] is going to be used in the experiments section

Theorem 1: Let $\mathbf{\Sigma}=(\mathbf{E}, \mathbf{A}, \mathbf{B}, \mathbf{C}, \mathbf{D}) \in \boldsymbol{\Sigma}_{n, p, m}^{-}$and $\sigma_{1}$ be the largest singular value of this system. Take $\gamma>0$ so that $\gamma \geqslant \sigma_{1}$ and also consider the $\mathcal{P}$ and $\mathcal{Q}$ Gramians as defined in Proposition 1. Compute the following matrices

$$
\begin{aligned}
& \mathbf{R}_{\boldsymbol{\Sigma}, \gamma}=\mathcal{Q} \mathbf{E} \mathcal{P} \mathbf{E}^{T}-\gamma^{2} I, \mathbf{E}_{\boldsymbol{\Sigma}, \gamma}=\mathbf{E}^{T} \mathbf{R}_{\boldsymbol{\Sigma}, \gamma}, \mathbf{B}_{\boldsymbol{\Sigma}, \gamma}=\mathbf{E}^{T} \mathcal{Q} \mathbf{B}, \\
& \mathbf{C}_{\boldsymbol{\Sigma}, \gamma}=\mathbf{C} \mathcal{P} \mathbf{E}^{T}, \mathbf{A}_{\boldsymbol{\Sigma}, \gamma}=-\mathbf{A}^{T} \mathbf{R}_{\boldsymbol{\Sigma}, \gamma}-\mathbf{C}^{T} \mathbf{C}_{\boldsymbol{\Sigma}, \gamma} .
\end{aligned}
$$

If $\left(\mathbf{E}_{\boldsymbol{\Sigma}, \gamma}, \mathbf{A}_{\boldsymbol{\Sigma}, \gamma}\right)$ is regular (for $\left.\gamma>\sigma_{1}\right)$, then $\boldsymbol{\Sigma}_{\gamma}:=\left(\mathbf{E}_{\boldsymbol{\Sigma}, \gamma}\right.$, $\left.\mathbf{A}_{\boldsymbol{\Sigma}, \gamma}, \mathbf{B}_{\boldsymbol{\Sigma}, \gamma}, \mathbf{C}_{\boldsymbol{\Sigma}, \gamma}, \mathbf{D}_{\boldsymbol{\Sigma}, \gamma}\right) \in \mathbf{\Sigma}_{n, p, m}^{+}$and the inequality holds: $\sigma_{1} \leqslant\left\|\mathbf{H}_{\boldsymbol{\Sigma}}-\mathbf{H}_{\boldsymbol{\Sigma}_{\gamma}}\right\|_{\infty} \leqslant \gamma$.

The main result is presented in the following theorem which was first stated in [16], i.e., 
Theorem 2: Consider $\boldsymbol{\Sigma} \in \boldsymbol{\Sigma}_{n, p, m}^{0}$ which can be decomposed as stated in Lemma 1 into $\boldsymbol{\Sigma}_{-}$and $\boldsymbol{\Sigma}_{+}$. Let $\sigma_{1}$ the

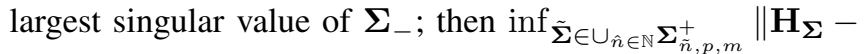
$\mathbf{H}_{\tilde{\boldsymbol{\Sigma}}} \|_{\infty}=\sigma_{1}$ follows. Let $r=\operatorname{rank}\left(\mathbf{A}_{\boldsymbol{\Sigma}_{-}, \sigma_{1}}\right)$ and $\mathbf{V}, \mathbf{W} \in$ $\mathbb{R}^{n_{-} \times r}$ such that $\mathbf{W}^{T} \mathbf{A}_{\boldsymbol{\Sigma}_{-}, \sigma_{1}} \mathbf{V}$ is regular. Then, $\left(\mathrm{AP}_{\infty}\right)$ is solved by $\boldsymbol{\Sigma}_{+} \oplus\left(\mathbf{W}^{T} \boldsymbol{\Sigma}_{-\sigma_{1}} \mathbf{V}\right)$.

Hence, when the dilation coefficient $\gamma$ coincides with the value of the largest singular value $\sigma_{1}$, one obtains an optimal approximate system in $\mathcal{R} \mathcal{H}_{\infty}$. When increasing $\gamma$ beyond the threshold $\sigma_{1}$, sub-optimal systems are instead computed. As shown in [13], in some cases such systems could be used instead of the optimal one. More precisely, the transfer function of reduced sub-optimal systems may match the initial transfer function at $\infty$, while this property might not be satisfied when $\gamma=\sigma_{1}$.

\section{DATA-DRIVEN MODELING METHODS}

\section{A. The Loewner framework ( $L F)$}

In this section, we present a brief overview of the Loewner framework for the multi-input multi-output (MIMO) case. For a complete description, we refer the reader to [6], and to [1] for insight in the rectangular case. LF is an approach based on the analysis of Loewner matrices. It is a data-driven method aimed at building a rational descriptor LTI dynamical model with transfer function $\mathbf{H}_{M}: \mathbb{C} \rightarrow \mathbb{C}^{p \times m}$ of dimension $M$ which interpolates given complex data. Here, the data are generated by a model with transfer function $\mathbf{H}: \mathbb{C} \rightarrow \mathbb{C}^{p \times m}$. Let the left (or row) data be given together with the right (or column) data, as below

$$
\left.\begin{array}{c}
\left(\mu_{j}, \mathbf{l}_{j}^{H}, \mathbf{v}_{j}^{H}\right) \\
\text { for } j=1, \ldots, M
\end{array}\right\} \text { and }\left\{\begin{array}{c}
\left(\lambda_{i}, \mathbf{r}_{i}, \mathbf{w}_{i}\right) \\
\text { for } i=1, \ldots, M
\end{array},\right.
$$

where $\mathbf{v}_{j}^{H}=\mathbf{l}_{j}^{H} \mathbf{H}\left(\mu_{j}\right)$ and $\mathbf{w}_{i}=\mathbf{H}\left(\lambda_{i}\right) \mathbf{r}_{i}$, with $\mathbf{l}_{j} \in \mathbb{C}^{p \times 1}$, $\mathbf{r}_{i} \in \mathbb{C}^{m \times 1}, \mathbf{v}_{j} \in \mathbb{C}^{m \times 1}$ and $\mathbf{w}_{i} \in \mathbb{C}^{p \times 1}$. In addition, the set of distinct interpolation points $\left\{z_{k}\right\}_{k=1}^{2 M} \subset \mathbb{C}$ is split up into two equal subsets $\left(\lambda_{i}, \mu_{j} \in \mathbb{C}\right)$, i.e.

$$
\left\{z_{k}\right\}_{k=1}^{2 M}=\left\{\mu_{j}\right\}_{j=1}^{M} \cup\left\{\lambda_{i}\right\}_{i=1}^{M} .
$$

The method then consists in building the Loewner matrix $\mathbb{L} \in$ $\mathbb{C}^{M \times M}$ and shifted Loewner matrix $\mathbb{L}_{s} \in \mathbb{C}^{M \times M}$ defined as follows, for $i=1, \ldots, M$ and $j=1, \ldots, M$ :

$$
\begin{aligned}
{[\mathbb{L}]_{j, i} } & =\frac{\mathbf{v}_{j}^{H} \mathbf{r}_{i}-\mathbf{l}_{j}^{H} \mathbf{w}_{i}}{\mu_{j}-\lambda_{i}}=\frac{\mathbf{l}_{j}^{H}\left(\mathbf{H}\left(\mu_{j}\right)-\mathbf{H}\left(\lambda_{i}\right)\right) \mathbf{r}_{i}}{\mu_{j}-\lambda_{i}}, \\
{\left[\mathbb{L}_{s}\right]_{j, i} } & =\frac{\mu_{j} \mathbf{v}_{j}^{H} \mathbf{r}_{i}-\lambda_{i} \mathbf{l}_{j}^{H} \mathbf{w}_{i}}{\mu_{j}-\lambda_{i}}=\frac{\mathbf{l}_{j}^{H}\left(\mu_{j} \mathbf{H}\left(\mu_{j}\right)-\lambda_{i} \mathbf{H}\left(\lambda_{i}\right)\right) \mathbf{r}_{i}}{\mu_{j}-\lambda_{i}} .
\end{aligned}
$$

Additionally, introduce the following matrices

$$
\mathbb{V}=\left[\begin{array}{lll}
\mathbf{v}_{1} & \cdots & \mathbf{v}_{M}
\end{array}\right]^{T}, \mathbb{W}=\left[\begin{array}{lll}
\mathbf{w}_{1} & \cdots & \mathbf{w}_{M}
\end{array}\right],
$$

where for all $j, i=1, \ldots, M$ we have that

$$
\mathbf{v}_{j}^{H}=\mathbf{l}_{j}^{H} \mathbf{H}\left(\mu_{j}\right), \quad \text { and } \quad \mathbf{w}_{i}=\mathbf{H}\left(\lambda_{i}\right) \mathbf{r}_{i} .
$$

Finally, let

$$
\begin{aligned}
\mathbf{M} & =\operatorname{diag}\left(\mu_{1}, \cdots, \mu_{M}\right), \quad \boldsymbol{\Lambda}=\operatorname{diag}\left(\lambda_{1}, \cdots, \lambda_{M}\right), \\
\mathbf{L} & =\left[\begin{array}{lll}
\mathbf{l}_{1} & \cdots & \mathbf{l}_{M}
\end{array}\right], \quad \mathbf{R}=\left[\begin{array}{lll}
\mathbf{r}_{1} & \cdots & \mathbf{r}_{M}
\end{array}\right] .
\end{aligned}
$$

The following Sylvester equations are hence satisfied by the Loewner matrix $\mathbb{L}$, and by the shifted Loewner matrix $\mathbb{L}_{s}$

$$
\begin{aligned}
\mathbf{M} \mathbb{L}-\mathbb{L} \boldsymbol{\Lambda} & =\mathbb{V} \mathbf{R}-\mathbf{L} \mathbb{W}, \\
\mathbf{M} \mathbb{L}_{s}-\mathbb{L}_{s} \boldsymbol{\Lambda} & =\mathbf{M} \mathbb{V} \mathbf{R}-\mathbf{L} \mathbb{W} \boldsymbol{\Lambda} .
\end{aligned}
$$

Then, the model $\mathbf{H}_{M}$ is given by the descriptor realization,

$$
\boldsymbol{\Sigma}_{M}:\left\{\begin{aligned}
\mathbf{E}_{M} \dot{\mathbf{x}}(t) & =\mathbf{A}_{M} \mathbf{x}(t)+\mathbf{B}_{M} \mathbf{u}(t) \\
\mathbf{y}(t) & =\mathbf{C}_{M} \mathbf{x}(t)
\end{aligned}\right.
$$

where $\mathbf{E}_{M}=-\mathbb{L}, \mathbf{A}_{M}=-\mathbb{L}_{s}, \mathbf{B}_{M}=\mathbb{V}$ and $\mathbf{C}_{M}=\mathbb{W}$. The associated transfer function given by

$$
\mathbf{H}_{M}(s)=\mathbf{C}_{M}\left(s \mathbf{E}_{M}-\mathbf{A}_{M}\right)^{-1} \mathbf{B}_{M},
$$

hence interpolates $\mathbf{H}$ at the given driving frequencies and directions defined in (5), i.e. satisfies the conditions

$$
\begin{aligned}
\mathbf{l}_{j}^{H} \mathbf{H}_{M}\left(\mu_{j}\right) & =\mathbf{l}_{j}^{H} \mathbf{H}\left(\mu_{j}\right), \\
\mathbf{H}_{M}\left(\lambda_{i}\right) \mathbf{r}_{i} & =\mathbf{H}\left(\lambda_{i}\right) \mathbf{r}_{i} .
\end{aligned}
$$

Assuming that the number $2 M$ of available data is large enough, then it was shown in [17] that a minimal model $\mathbf{H}_{n}$ of dimension $n<M$ (that still interpolates the data) can be computed with a projection of (12) provided that the following holds for $k=1, \ldots, 2 M$

$\operatorname{rank}\left(z_{k} \mathbb{L}-\mathbb{L}_{s}\right)=\operatorname{rank}\left(\left[\mathbb{L}, \mathbb{L}_{s}\right]\right)=\operatorname{rank}\left(\left[\mathbb{L}^{H}, \mathbb{L}_{s}{ }^{H}\right]^{H}\right)=n$,

where $z_{k}$ are as in (6). In that case, let $\mathbf{Y} \in \mathbb{C}^{M \times n}$ be the matrix containing the first $n$ left singular vectors of $\left[\mathbb{L}, \mathbb{L}_{s}\right]$ and $\mathbf{X} \in \mathbb{C}^{M \times n}$ the matrix containing the first $n$ right singular vectors of $\left[\mathbb{L}^{H}, \mathbb{L}_{s}^{H}\right]^{H}$. Then,

$$
\begin{aligned}
& \mathbf{E}_{n}=\mathbf{Y}^{H} \mathbf{E}_{M} \mathbf{X}, \mathbf{A}_{n}=\mathbf{Y}^{H} \mathbf{A}_{M} \mathbf{X}, \\
& \mathbf{B}_{n}=\mathbf{Y}^{H} \mathbf{B}_{M}, \mathbf{C}_{n}=\mathbf{C}_{M} \mathbf{X},
\end{aligned}
$$

is a realization of the model $\mathbf{H}_{n}$, given as,

$$
\mathbf{H}_{n}(s)=\mathbf{C}_{n}\left(s \mathbf{E}_{n}-\mathbf{A}_{n}\right)^{-1} \mathbf{B}_{n},
$$

with the same structure as (13), encoding a minimal McMillan degree equal to $\operatorname{rank}(\mathbb{L})$. The quintuple $\mathcal{S}_{n}$ : $\left(\mathbf{E}_{n}, \mathbf{A}_{n}, \mathbf{B}_{n}, \mathbf{C}_{n}, \mathbf{0}\right)$ is a descriptor realization of $\mathbf{H}_{n}$. Note that if $n$ in (15) is greater than $\operatorname{rank}(\mathbb{L})$, then $\mathbf{H}_{n}$ can either have a direct-feedthrough term or a polynomial part. Finally, the number $n$ of singular vectors composing $\mathbf{Y}$ and $\mathbf{X}$ used to project the system $\mathbf{H}_{n}$ in (16) may be decreased to $r<n$ at the cost of imposing an approximate interpolation of data, leading to the reduced model $r$-th order rational model. This allows a trade-off between complexity of the resulting model and accuracy of the interpolation.

\section{B. The AAA algorithm}

The AAA algorithm, originally proposed in [18], represents an adaptive extension of the interpolation-based method introduced in [2]. It is a robust, fast and effective method that was mainly used for scalar rational interpolation applications. AAA is a multi-step algorithm, that computes at step $\ell$ a rational approximant of order $(\ell, \ell)$ in barycentric representation. In this note we discuss a slightly modified version from that in [18], in the sense that the approximant at step $\ell$ 
is strictly proper, i.e. of order $(\ell-1, \ell)$. Additionally, as for the Loewner method, we will enforce real-valued models. Finally, we restrict the presentation to the SISO case (the MIMO case was recently proposed in [14]).

As in (6), we consider at step $\ell \geq 1$ the data splitting:

$$
\begin{aligned}
\text { data points : } & \left\{z_{k}\right\}_{k=1}^{2 M}=\left\{\nu_{j}\right\}_{j=1}^{\ell} \cup\left\{\eta_{i}\right\}_{i=1}^{2 M-\ell}, \\
\text { data values : } & \left\{f_{k}\right\}_{k=1}^{2 M}=\left\{h_{j}\right\}_{j=1}^{\ell} \cup\left\{g_{i}\right\}_{i=1}^{2 M-\ell} .
\end{aligned}
$$

Note that in the representation given in (18), the values $f_{k}$ represent the measurements evaluated at the points $z_{k}$, while $h_{j}$ and $g_{i}$ are the ones evaluated at $\nu_{j}$, and respectively at $\eta_{i}$. The rational interpolant $\mathbf{H}_{\ell}$, obtained after $\ell$ iterations of the AAA algorithm, has the form

$$
\mathbf{H}_{\ell}(\xi)=\frac{\sum_{j=1}^{\ell} \frac{\alpha_{j}^{(\ell)} h_{j}}{\xi-\nu_{j}}}{1+\sum_{j=1}^{\ell} \frac{\alpha_{j}^{(\ell)}}{\xi-\nu_{j}}},
$$

with nonzero barycentric weights $\alpha_{j}^{(\ell)} \in \mathbb{C}$, pairwise distinct support points $\nu_{j} \in \mathbb{C}$, and function values $h_{j}$. Based on the representation in (19), interpolation is enforced at the first subset of data points $\left\{\nu_{j}\right\}_{j=1}^{\ell}$, i.e. $\mathbf{H}_{\ell}\left(\nu_{j}\right)=h_{j}$ for $1 \leq j \leq$ $\ell$. In order to completely determine the approximant $\mathbf{H}_{\ell}$, one needs to also find the barycentric weights $\alpha_{1}^{(\ell)}, \ldots, \alpha_{\ell}^{(\ell)}$. This is done by solving a least squares problem. Finally, the next support point is chosen by means of a greedy selection.

Based on the representation of the transfer function at step $\ell$ in (19), one can formulate the minimization problem as

$$
\begin{array}{r}
\min _{\alpha_{1}^{(\ell)}, \ldots, \alpha_{\ell}^{(\ell)}} \sum_{k=1}^{2 M}\left(\mathbf{H}_{\ell}\left(z_{k}\right)-f_{k}\right)^{2} \\
\Leftrightarrow \min \sum_{k=1}^{2 M}\left(\frac{\sum_{j=1}^{\ell} \frac{\alpha_{j}^{(\ell)}\left(f_{k}-h_{j}\right)}{z_{k}-\nu_{j}}+f_{k}}{1+\sum_{j=1}^{\ell} \frac{\alpha_{j}^{(\ell)}}{z_{k}-\nu_{j}}}\right)^{2} .
\end{array}
$$

Instead of solving the nonlinear problem in (20), one solves a linearized problem derived from it, as further explained in step 5 of the algoritm below.

Let $\tau$ be the desired tolerance for data approximation and let $r$ denote the target dimension. The modified AAA algorithm can be summarized as follows:

\section{1) Initialization step}

Set $\ell=0, \Omega^{(0)}:=\Omega$, and $\mathbf{H}_{0}(\xi)=\frac{1}{2 M} \sum_{k=1}^{2 M} f_{k}$.

2) While $\max _{1 \leq k \leq 2 M}\left|f_{k}-\mathbf{H}_{\ell}\left(z_{k}\right)\right|>\tau$ and $\ell<r$

3) do $\ell=\ell+1$.

4) Find $\nu_{\ell} \in \Omega^{(\ell-1)}$ as $\nu_{\ell}=\underset{1 \leq k \leq 2 M}{\operatorname{argmax}}\left|f_{k}-\mathbf{H}_{\ell-1}\left(z_{k}\right)\right|$, with $\mathbf{H}_{\ell}(s)$ as in (19). Set $h^{\ell}:=\mathbf{H}\left(\nu_{\ell}\right)$, and also $\Omega^{(\ell)}:=\Omega^{(\ell-1)} \backslash\left\{\nu_{\ell}\right\}$.

5) Compute weights $\alpha_{1}^{(\ell)}, \ldots, \alpha_{\ell}^{(\ell)}$ to minimize the deviation in the measurements, i.e. solve the problem

$$
\min _{\boldsymbol{\alpha}^{(\ell)}} \sum_{k=1}^{2 M} \sum_{j=1}^{\ell}\left(\frac{\left(f_{k}-h_{j}\right) \alpha_{j}^{(\ell)}}{z_{k}-\nu_{j}}+f_{k}\right)^{2} \Leftrightarrow\left\|\mathbb{L} \boldsymbol{\alpha}^{(\ell)}+\mathbf{f}\right\|_{2}^{2},
$$

where $\mathbb{L} \in \mathbb{C}^{2 M \times \ell}$ with $\mathbb{L}_{k, j}=\frac{f_{k}-h_{j}}{z_{k}-\nu_{j}}$ is a Loewner matrix, while $\boldsymbol{\alpha}^{(\ell)} \in \mathbb{C}^{\ell}$, and $\mathbf{f} \in \mathbb{C}^{2 M}$.

6) Compute the solution to (21) as $\boldsymbol{\alpha}^{(\ell)}=-\mathbb{L}^{\#} \mathbf{f}$, where $\mathbb{L}^{\#} \in \mathbb{C}^{\ell \times 2 M}$ is the pseudo-inverse of matrix $\mathbb{L}$. end

Note that a realization $\boldsymbol{\Sigma}_{r}^{\mathrm{AAA}}:\left(\mathbf{I}_{r}, \tilde{\mathbf{A}}_{r}, \tilde{\mathbf{B}}_{r}, \tilde{\mathbf{C}}_{r}, 0\right)$ of an order $(r-1, r)$ AAA model can be expressed as follows

$$
\begin{aligned}
\tilde{\mathbf{A}}_{r} & =\operatorname{diag}\left(\nu_{1}, \ldots, \nu_{r}\right)-\mathbf{B}_{r} \mathbf{e}_{r}^{T}, \\
\tilde{\mathbf{B}}_{r} & =\left[\begin{array}{lll}
\alpha_{1}^{(r)} & \ldots & \alpha_{r}^{(r)}
\end{array}\right]^{T}, \quad \tilde{\mathbf{C}}_{r}=\left[\begin{array}{lll}
h_{1} & \ldots & h_{r}
\end{array}\right],
\end{aligned}
$$

where $\mathbf{e}_{r}=\left[\begin{array}{lll}1 & \cdots & 1\end{array}\right]^{T}$ and $\tilde{\mathbf{A}}_{r}$ is a rank-1 perturbation of the diagonal matrix composed of the support points.

\section{NUMERICAL EXAMPLES}

In the numerical test cases presented in this section we will be applying the Loewner method and AAA algorithm, as presented in Section III-A and Section III-B respectively. Note that Loewner-K and AAA-K correspond to the postprocessed systems computed with Köhler's method summarized in Section II-B (for the $\mathcal{R} \mathcal{H}_{\infty}$ space $^{1}$ ).

\section{A. A toy example}

Assume that the transfer function is given by

$$
\mathbf{H}(s)=\underbrace{\frac{1}{s+3}}_{\mathbf{H}_{-}(s)}+\underbrace{\frac{2}{s-2}-\frac{1}{s-1}}_{\mathbf{H}_{+}(s)} .
$$

Above, $\mathbf{H}_{-}(s)=\frac{1}{s+3}$ is the stable part, while $\mathbf{H}_{+}(s)=$ $\frac{2}{s-2}-\frac{1}{s-1}$ is the antistable one of the original transfer function. The two singular values of the antistable part are equal, e.g. $\sigma_{1}=\sigma_{2}=\frac{1}{6}$. Below we compute the optimal approximation in $\mathcal{R H}_{2}$, together with the optimal and suboptimal approximations in $\mathcal{R H}_{\infty}$ (in this order).

1) $\mathbf{H}_{-}(s)=\frac{1}{s+3}, \quad\left\|\boldsymbol{\Sigma}-\boldsymbol{\Sigma}_{-}\right\|_{\mathcal{H}_{\infty}}=\frac{1}{3}$,

2) $\mathbf{H}_{\text {opt }}(s)=\frac{1}{s+3}-\frac{1}{6}, \quad\left\|\boldsymbol{\Sigma}-\boldsymbol{\Sigma}_{\text {opt }}\right\|_{\mathcal{H}_{\infty}}=\frac{1}{6}$,

3) $\mathbf{H}_{\text {sopt }}^{\gamma}(s)=\frac{1}{s+3}-\frac{s}{6\left(8 s^{2}+25 s+16\right)}, \quad\left\|\boldsymbol{\Sigma}-\boldsymbol{\Sigma}_{\text {sopt }}\right\|_{\mathcal{H}_{\infty}}=$ $\frac{49}{150}$, for $\gamma=\frac{7}{6}$.

The sub-optimal approximation $\mathbf{H}_{\text {sopt }}^{\gamma}$ depends on the free parameter $\gamma \geq \sigma_{1}$; also, note that $\mathbf{H}_{o p t}=\mathbf{H}_{\text {sopt }}^{\sigma_{1}}$ holds true.

\section{B. A model for a clamped beam}

We analyze the clamped beam model from the collection of model reduction benchmarks in [10]. The model is an LTI system with $n=348$ state variables, one input and one output, and is obtained by spatial discretization of a partial differential equation. The control input is represented by the force applied to the structure at the free end, and the output is the resulting displacement. It should be noted that the original model is stable, i.e. all poles are in $\mathbb{C}_{-}$.

Choose 100 logarithmically-spaced points in the interval $\left[10^{-2}, 10^{2}\right] \cdot \imath$ together with the complex conjugate values. The evaluation of the transfer function of the model at these frequencies is displayed in Fig. 1.

\footnotetext{
${ }^{1}$ Note that the $\mathcal{R} \mathcal{H}_{2}$ case simply consists in discarding the unstable part of the model.
} 


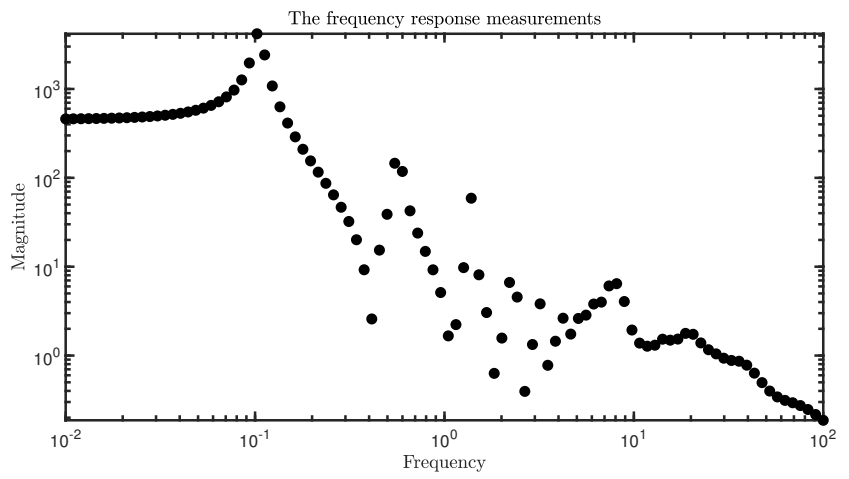

Fig. 1. The data measurement for the beam example.

For this particular data set, we apply both the Loewner and AAA methods introduced in Section III for different reduction orders $r=2 \ldots 50$. For all these reduced-order models, we compute the number of antistable poles and we display the actual values in Fig. 2.

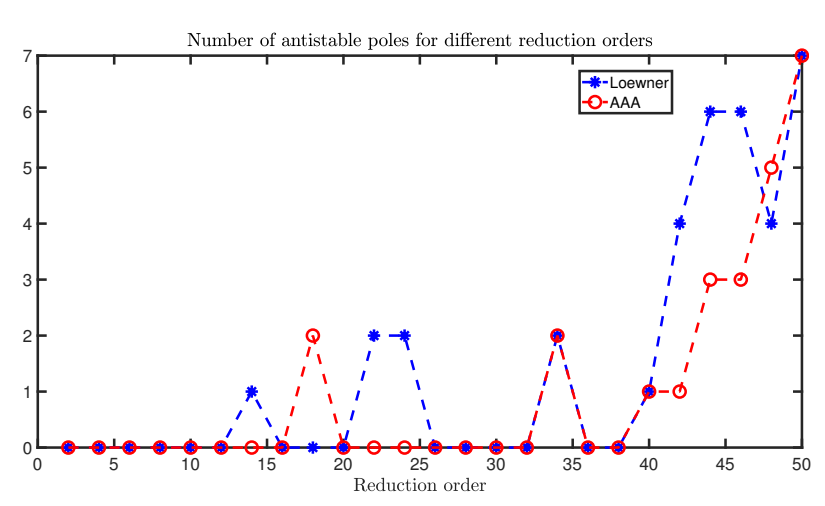

Fig. 2. The number of antistable poles for different reduction orders.

Next, for all reduction orders $r \in[2,50]$ that yield unstable models, we apply the post-processing method presented in Section II-B to the computed reduced models. The maximum absolute approximation errors are displayed in Fig. 3. More precisely, for each order $r$, we compute the maximum deviation between the original data and the evaluation of the reduced-order transfer functions on the data set. We display results corresponding to the original methods and also to the post-processed methods. It is to be noted that in the case of the latter, the approximation errors indeed increase (this is the price to pay for enforcing stability).

Finally, we compute the $\mathcal{H}_{2}$ and $\mathcal{H}_{\infty}$ relative approximation errors for each order $r=2 \ldots 50$. Note that, whenever an unstable model is encountered, the post-processing method is applied. The results are displayed in Fig. 4.

By inspecting Fig. 2, one can notice that from $r=2$ up to $r=40$, only one or two antistable eigenvalues appear in the approximated models at some sparse scattered $r$ order cases. Therefore, the post-stabilization is not always needed, and when needed, it does not gravely impact the mismatch error, as illustrated in Fig. 3. On the other hand, when $r>40$, the number of antistable (and hence non physical) eigenvalues increases. At the same time, the poststabilization results show that mismatch errors increase for

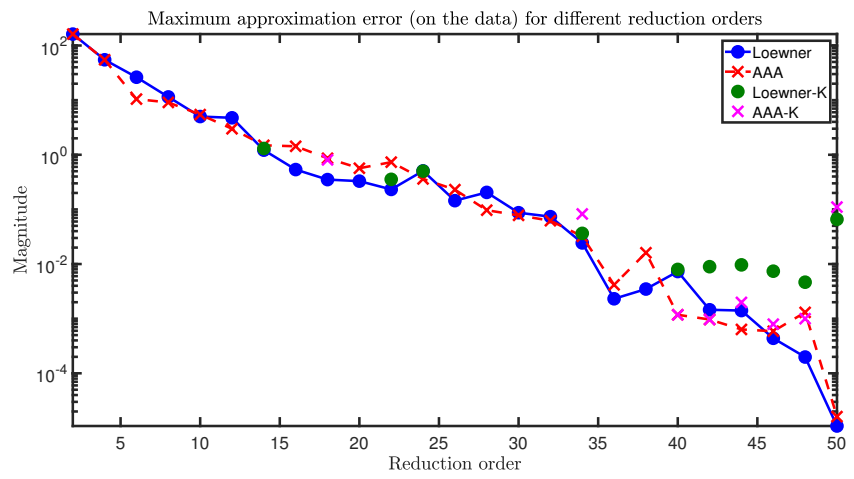

Fig. 3. The maximum approximation errors on the data.
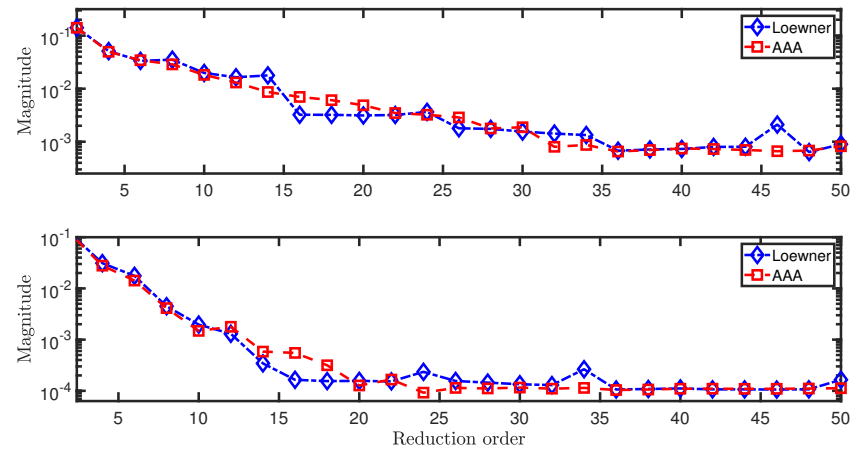

Fig. 4. The $\mathcal{H}_{2}$ (up) and $\mathcal{H}_{\infty}$ (down) relative approximation errors.

orders between 40 and 50, as it can be observed again in Fig. 3. These phenomena can be connected to the singular values decay of the Loewner pencil. Indeed, after $r>40$ the singular values of the pencil approach machine precision. As a consequence, the additional dynamics in the approximated model can be considered not as relevant. As stated in chapter 5 of [20], from a stable model $\mathbf{H}$ it is always possible to find an approximated model $\hat{\mathbf{H}}$ that is unstable and that minimises the $\mathcal{L}_{2}$ mismatch problem. This is precisely what is observed for this numerical example.

\section{The simplified 1D St Venant PDE equation}

1) Model description: The so-called Saint-Venant equations are largely used in the hydraulic domain to model the dynamics of an open channel flow. These equations consist of two nonlinear hyperbolic PDEs. In the considered example, under mild simplifying assumptions detailed in [11], the St Venant PDE equations describing the height variation $h$ of the river as a function of the inflow $q_{i}$ and outflow $q_{o}$ variations, at location $x\left(x \in[0 L], L \in \mathbb{R}_{+}\right)$, obtained around some flow and height linearization point, can be formulated as follows:

$$
\begin{aligned}
h(x, s) & =\mathbf{G}_{\mathbf{i}}(x, s) q_{i}(s)-\mathbf{G}_{\mathbf{o}}(x, s) q_{o}(s) \\
& =\mathbf{H}(x, s) \mathbf{u}(s)
\end{aligned}
$$

The irrational functions $\mathbf{G}_{i}$ and $\mathbf{G}_{o}$ are expressed as below

$$
\begin{aligned}
\mathbf{G}_{\mathbf{i}}(x, s) & =\frac{\lambda_{1}(s) e^{\lambda_{2}(s) L+\lambda_{1}(s) x}-\lambda_{2}(s) e^{\lambda_{1}(s) L+\lambda_{2}(s) x}}{B_{0} s\left(e^{\lambda_{1}(s) L}-e^{\lambda_{2}(s) L}\right)} \\
\mathbf{G}_{\mathbf{o}}(x, s) & =\frac{\lambda_{1}(s) e^{\lambda_{1}(s) x}-\lambda_{2}(s) e^{\lambda_{2}(s) x}}{B_{0} s\left(e^{\lambda_{1}(s) L}-e^{\lambda_{2}(s) L}\right)}
\end{aligned}
$$


All numerical values for the configuration $x=x_{m}=5600$, together with a detailed description, can be found in $[11]^{2}$. Moreover, $\mathbf{H} \in \mathbb{C}^{1 \times 2}$ is the associated transfer function mapping $\mathbf{u}=\left[\begin{array}{ll}q_{i} & q_{o}\end{array}\right]$ to $h$. Because of the relative complex form of (24), it is hard to simulate and to analyze due to the difficulty to recover the time-domain trajectories in response to some input. By sampling the first input along the imaginary axis, e.g. 600 logarithmically-spaced points in $\left[10^{-4}, 10^{-1.5}\right] \cdot \imath$, Fig. 5 data are collected ${ }^{3}$.

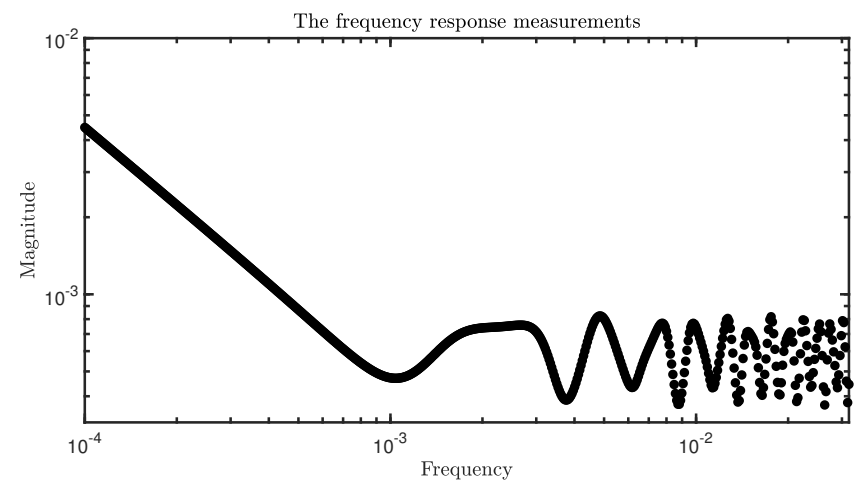

Fig. 5. Data for the simplified 1D St Venant PDE equation.

2) Model preliminary remarks and analysis: At this stage, one may assume some specific properties of the use-case under consideration. Among them, the first remark is that the system embeds delay terms both in the numerator (inputs) and in the denominator (dynamics), as well as a singularity at zero. Therefore, $\mathbf{H}$ is not rational and $\mathbf{H} \in \mathcal{L}_{\infty}$ or $\mathcal{L}_{2}$ (and not in $\mathcal{H}_{\infty}$ or $\mathcal{H}_{2}$ ). Moreover, the model has an infinite number of singularities due to the harmonic functions in the denominator. Finally, as illustrated in Figure 5, the gain in high frequency oscillates around some constant term, therefore $\mathbf{H} \in \mathcal{L}_{\infty}$. As a consequence of this, when performing approximation either in the Loewner or the AAA frameworks, according to the selected frequency grid, one may end up with a high frequency undamped (antistable) pair of poles. This probably does not have a clear physical meaning. Moreover, the singularity at zero may be an issue when enforcing the stability property.

In order to attenuate this behaviour, which may also cause certain numerical issues, one solution is to consider instead the following shifted model:

$$
\tilde{\mathbf{H}}(s)=\mathbf{H}(s) \mathbf{F}(s),
$$

where $\mathbf{F}(s)=\frac{s}{(s+\alpha)(s+\beta)}$ and $(0>\alpha>\beta)^{4}$. By using this transformation, one can observe that the singularity at zero is cancelled and that the oscillatory behaviour at infinity now decays to zero. Then, $\tilde{\mathbf{H}} \in \mathcal{H}_{2}$ while $\mathbf{H} \in \mathcal{L}_{\infty}$. This slight modification is useful for both numerical and practical issues. Indeed, by using this transformation, we

\footnotetext{
${ }^{2}$ The Matlab benchmark is also available in the MOR Wiki webpage, in the "Hydro-Electric Open Channel" category.

${ }^{3}$ From now on, we will get rid of the $x$ term in the transfer (23) and consider $\mathbf{H}(s)$ only.

${ }^{4}$ In the remaining of the section we consider $\alpha=10^{-2}$ and $\beta=10^{-3}$.
}

use some a priori known physical properties of the system in order to improve the identification process. Consequently, the post-treatment for stability enforcement is now meaningful, even for the case of unstable systems (often encountered in engineering applications).

For the data set corresponding to samples of the function in (25), we apply both the Loewner and AAA methods introduced in Section III. We vary the reduction orders $r$ in the interval $[2,54]$. For all reduced-order models, display the number of antistable poles in Fig. 6.

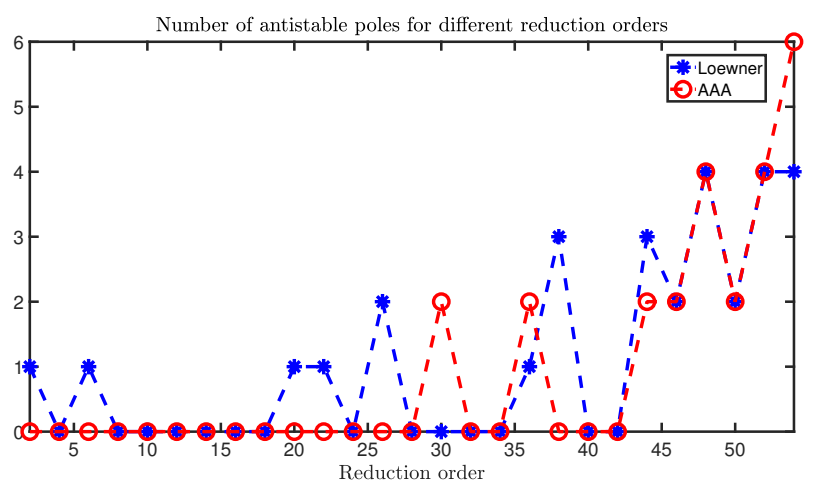

Fig. 6. The number of antistable poles for different reduction orders.

As done for the beam example in Section IV-B, we apply the post-processing method presented in Section II-B to the computed reduced models. This is performed for all reduction orders $r \in[2,54]$ that yield unstable models. The next step is to convert the response of the models by multiplying its transfer function with $1 / \mathbf{F}(s)$. Hence, by doing this, we obtain models that fit very well the original data associated to $\mathbf{H}(s)$, displayed in Fig. 5. Furthermore, for each order $r$, we compute the maximum deviation between the original data and the evaluation of the reduced-order transfer functions on the data set. We display results corresponding to the original methods and also to the post-processed methods in Fig. 7.

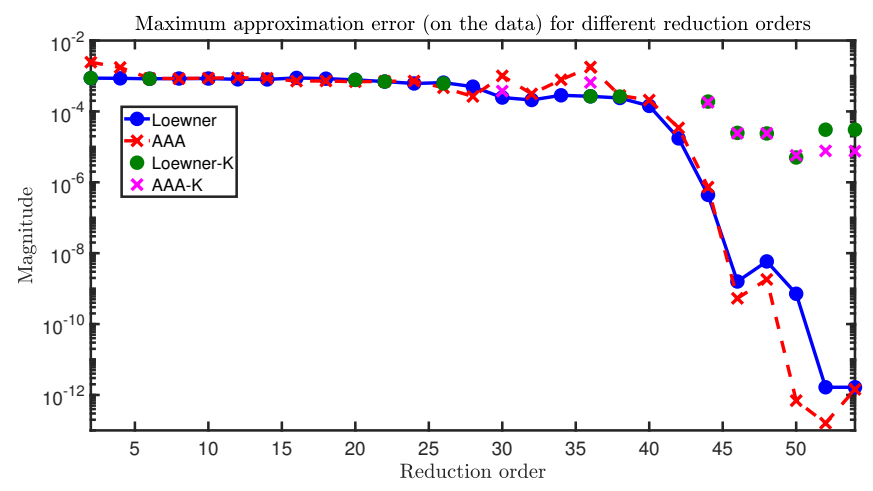

Fig. 7. Maximum approximation errors on the data.

For the next experiment, fix the reduction order to be $r=$ 50 for both Loewner-K and AAA-K post-processed models. We observe very good approximation errors for the surrogate models (with all poles in $\mathbb{C}_{-}$and one pole at $s=0$ ), as displayed in Fig. 8.

Finally, the absolute approximation errors for each frequency values are displayed in Fig. 9. Again, as stated above, 


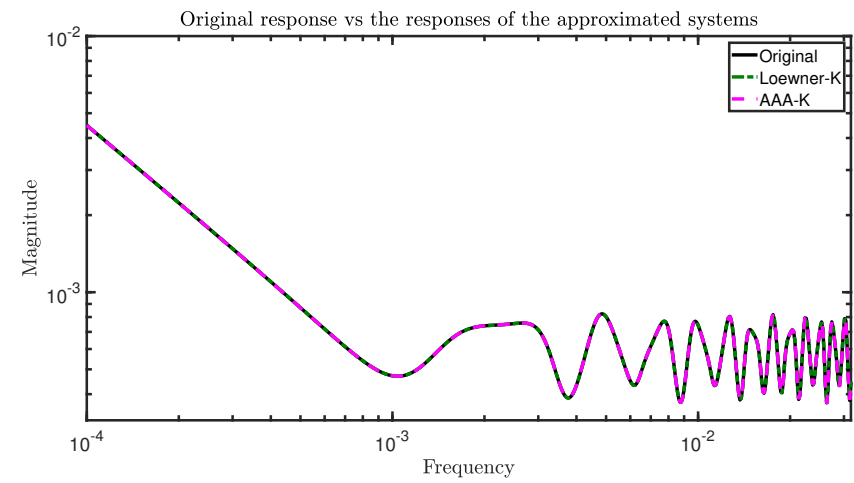

Fig. 8. The original response and that of the reduced-order models.

both methods perform really well in fitting the original response. The maximum error (on the data) is close to the value $10^{-5}$ for both methods, information which can be also deduced from Fig. 7 (when observing the values of Loewner$\mathrm{K}$ and AAA-K for $r=50)$.

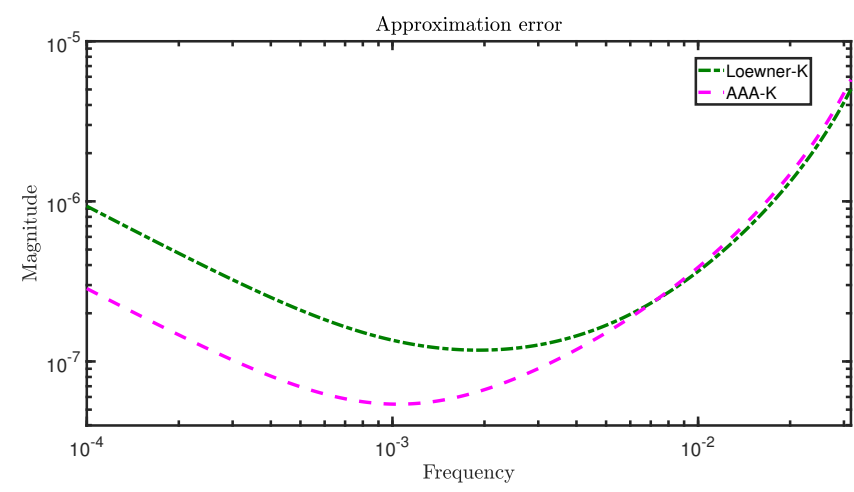

Fig. 9. The absolute approximation error on the data for both methods.

\section{CONCLUSION}

In this paper, we have proposed a detailed and comprehensive study on the application of the Loewner framework and of the AAA algorithm for data-driven approximation with stability enforcement. This work shows that even though these methods do not necessarily yield stable models, by applying an established post-processing method, we were able to construct stable reliable models. In some scenarios, as for the second numerical test case, it is required to perform additional processing of data in order to be able to retain certain important properties (such as the pole at 0 ). We have also shown that the approximation quality on the data, although decreases after performing the post-processing step, is still kept at an adequate level (as emphasized in the second numerical example). Future research includes estimating stability regions for delay systems by means of data-driven methods such as the ones covered here. Furthermore, one can pursue extending the approach for parametric LTI systems with different structures.

\section{REFERENCES}

[1] Antoulas, A.: The Loewner framework and transfer functions of singular/rectangular systems. App. Math. Letters 54, 36-47 (2016)
[2] Antoulas, A., Anderson, B.: On the scalar rational interpolation problem. IMA Journal of Mathematical Control and Information 3(23), 61-88 (1986)

[3] Antoulas, A.C.: Approximation of large-scale dynamical systems. SIAM, Philadelphia (2005)

[4] Antoulas, A.C., Beattie, C.A., Gugercin, S.: Interpolatory Methods for Model Reduction. SIAM, Philadelphia (2020)

[5] Antoulas, A.C., Gosea, I.V., Ionita, A.C.: Model reduction of bilinear systems in the Loewner framework. SIAM Journal on Scientific Computing 38(5), B889-B916 (2016)

[6] Antoulas, A.C., Lefteriu, S., Ionita, A.C.: A tutorial introduction to the Loewner framework for model reduction. In: Model Reduction and Approximation, chap. 8, pp. 335-376. SIAM (2017)

[7] Benner, P., Goyal, P., Van Dooren, P.: Identification of PortHamiltonian systems from frequency response data. Tech. rep., arXiv preprint available at https://arxiv.org/abs/1911.00080 (2019)

[8] Benner, P., Ohlberger, M., Cohen, A., Willcox, K.: Model Reduction and Approximation. SIAM, Philadelphia, PA (2017)

[9] Carracedo Rodriguez, A., Gugercin, S.: The p-AAA algorithm for data driven modeling of parametric dynamical systems. Tech. rep., arXiv preprint available at https://arxiv.org/abs/2003.06536 (2020)

[10] Chahlaoui, Y., Van Dooren, P.: Benchmark examples for model reduction of linear time-invariant dynamical systems. In: In: Benner P., Sorensen D.C., Mehrmann V. (eds), Dimension Reduction of LargeScale Systems, vol. 45, pp. 335-376. Springer, Berlin (2005)

[11] Dalmas, V., Robert, G., Poussot-Vassal, C., Pontes Duff, I., Seren, C.: From infinite dimensional modelling to parametric reduced order approximation: Application to open-channel flow for hydroelectricity. In: Proceedings of the 15th European Control Conference, pp. 19821987. Aalborg, Denmark (2016)

[12] Glover, K.: All optimal Hankel-norm approximations of linear multivariable systems and their $L_{\infty}$-error bounds. International Journal of Control 39, 1115 - 1193 (1984)

[13] Gosea, I.V., Antoulas, A.C.: Stability preserving post-processing methods applied in the Loewner framework. In: IEEE 20th Workshop on Signal and Power Integrity (SPI), Turin, Italy, May 8-11, pp. 1-4 (2016)

[14] Gosea, I.V., Güttel, S.: Algorithms for the rational approximation of matrix-valued functions. Tech. rep., arXiv preprint available at https://arxiv.org/abs/2003.06410 (2020)

[15] Ionutiu, R., Rommes, J., Antoulas, A.C.: Passivity preserving model reduction using dominant spectral zero interpolation. IEEE Transactions on Computer-Aided Design of Integrated Circuits and Systems 27(12), 2250-2263 (2008)

[16] Kohler, M.: On the closest stable descriptor system in the respective spaces $\mathcal{R H}_{2}$ and $\mathcal{R H}_{\infty}$. Linear Algebra and its Applications 443, 34-49 (2014)

[17] Mayo, A., Antoulas, A.: A framework for the solution of the generalized realization problem. Linear Algebra and Its Applications 425(23), 634-662 (2007)

[18] Nakatsukasa, Y., Sete, O., Trefethen, L.: The AAA algorithm for rational approximation. SIAM Journal on Scientific Computing 40(3), A1494-A1522 (2018)

[19] Peherstorfer, B., Gugercin, S., Willcox, K.: Data-driven reduced model construction with time-domain Loewner models. SIAM Journal on Scientific Computing 39(5), A2152-A2178 (2017)

[20] Poussot-Vassal, C.: Large-scale dynamical model approximation and its applications. HDR, habilitation thesis, Onera, INP Toulouse, Toulouse, France (2019)

[21] Poussot-Vassal, C., Kergus, P., Vuillemin, P.: Interpolation-based irrational model control design and stability analysis. In: Realization and Model Reduction of Dynamical Systems - A Festschrift in Honor of the 70th Birthday of Thanos Antoulas. Springer (2021). Accepted

[22] Poussot-Vassal, C., Quero, D., Vuillemin, P.: Data-driven approximation of a high fidelity gust-oriented flexible aircraft dynamical model. In: Proceedings of the IFAC Mathematical Modelling. Vienna, Austria (2018)

[23] Quero, D., Vuillemin, P., Poussot-Vassal, C.: A Generalized StateSpace Aeroservoelastic Model based on Tangential Interpolation. Aerospace 6(1) (2019)

[24] Sahouli, M., Dounavis, A.: Iterative Loewner matrix passivity correction technique. In: IEEE Electrical Design of Advanced Packaging and Systems (EDAPS), Shenzhen, China, pp. 1-3 (2020) 